\title{
Benefits of 1-Year Lifestyle Modification Program on Exercise Capacity and Diastolic Function Among Coronary Artery Disease Men With and Without Type 2 Diabetes
}

\author{
Marie-Eve Piché, MD, PhD, ${ }^{1,2}$ Paul Poirier, MD, PhD, ${ }^{1,3}$ André Marette, PhD, ${ }^{1}$ Patrick Mathieu, MD, ${ }^{1,2}$ \\ Valérie Lévesque, MSc, Karine Bibeau, BSc, Éric Larose, MD, ${ }^{1,2}$ and Jean-Pierre Després, PhD, FAHA, FIAS ${ }^{1,2}$
}

\begin{abstract}
Background: To assess the benefits of a 1-year lifestyle modification program on exercise capacity and diastolic function in men with left ventricular (LV) diastolic dysfunction (LVDD) and coronary artery disease (CAD), according to glucose tolerance status.

Methods: Fifty-three men (62 \pm 8 years; BMI: $\left.27.3 \pm 3.5 \mathrm{~kg} / \mathrm{m}^{2}\right)$ with LVDD and CAD were enrolled in a 1-year lifestyle modification program based on dietary management and increased physical activity. Patients were classified by using a 75 grams oral glucose tolerance test as having normal glucose tolerance $(n=16)$, prediabetes $(n=23)$, or type 2 diabetes mellitus (T2DM) $(n=14)$. Cardiac morphology and function, visceral fat, and cardiac fat depots were measured using magnetic resonance imaging, whereas exercise capacity [cardiorespiratory fitness $(\mathrm{CRF})]\left(\mathrm{VO}_{2 \text { peak }}\right)$ was assessed with a maximal treadmill test.

Results: The 1-year lifestyle modification program was associated with reductions in body weight, and visceral and cardiac fat levels (all $P<0.05)$. CRF increased by $13 \%\left(24.9 \pm 4.1\right.$ vs. $\left.28.2 \pm 4.8 \mathrm{~mL} \mathrm{O}_{2} / \mathrm{kg} / \mathrm{min}, P<0.0001\right)$. Moreover, half of patients (53\%) improved LV diastolic function in response to the lifestyle intervention. Multiple regression analyses revealed that age (partial $R^{2}=26.9, P<0.0001$ ) and presence of T2DM (partial $R^{2}=5.9, P=0.04$ ) were the stronger predictors of change in diastolic function, while favorable change in $\mathrm{LV}$ remodeling index was the best predictor of improvement in LV diastolic function after the lifestyle intervention $\left(R^{2}=21.9, P=0.002\right)$.

Conclusions: Irrespective of glucose tolerance status, a 1-year lifestyle modification program in men with LVDD and CAD is associated with significant improvements in exercise capacity and LV diastolic function in more than half of patients.
\end{abstract}

Keywords: lifestyle modification program, diastolic function, exercise capacity, coronary artery disease, cardiac fat depot, type 2 diabetes mellitus

\section{Introduction}

I eft ventricular (LV) diastolic dysfunction (LVDD) Lis a prevalent condition among patients with type 2 diabetes mellitus (T2DM) and coronary artery disease (CAD); it has been associated with increased morbidity and adverse cardiovascular outcomes. ${ }^{1}$ LVDD is also a major determinant of reduced functional capacity and exercise capacity among T2DM and CAD patients. ${ }^{2}$
In a recent meta-analysis on exercise training in heart failure with preserved ejection fraction ( $\mathrm{HFpEF})$, moderateintensity exercise training, either alone or in combination with strength training, demonstrates benefits on exercise capacity and LV diastolic function. ${ }^{3}$ Exercise training, as part of a comprehensive cardiac rehabilitation program, is effective at improving functional capacity and quality of life in CAD patients. ${ }^{4}$ Behind the exercise-related benefits, which are driven by cardiovascular risk factors modulation, ${ }^{5}$

\footnotetext{
${ }^{1}$ Department of Cardiology, Quebec Heart and Lung Institute, Quebec, Canada.

${ }^{2}$ Faculty of Medicine and ${ }^{3}$ Faculty of Pharmacy, Laval University, Quebec, Canada.
} 
evidence also suggests that the protective effect may be partly mediated through enhanced cardiac performance and LV diastolic function. ${ }^{6,7}$ Cardiac adaptations to exercisebased training interventions appear, however, to be variable, and determinants of a favorable response remain poorly understood in this high-risk population of patients.

Cardiac LV diastolic filling profile measured from cardiac magnetic resonance imaging (MRI) provides an alternate validated approach to echocardiographic measures of LV diastolic function. ${ }^{8-10}$ Using cardiac MRI, it has been demonstrated that LV peak filling rate (PFR) is reduced, and LV peak filling time is increased in patients with stable CAD. ${ }^{9}$ A reduced PFR has also been reported among men with metabolic syndrome. ${ }^{11}$ Using PFR normalized to LV stroke volume, Graca et al. have reported LVDD in patients with T2DM and CAD compared with those without CAD. ${ }^{12}$ Despite evidence of an abnormal LV diastolic filling profile associated with CAD, less is known about the influence of a lifestyle modification program on LV diastolic filling and diastolic function in patients with CAD and with varying levels of glucose tolerance.

The aim of this study was to assess the effect of a 1-year exercise-based training lifestyle intervention in addition to standard care on exercise capacity [cardiorespiratory fitness (CRF)] and on LV diastolic function in men with abnormal LV diastolic function and CAD, according to glucose tolerance status.

\section{Methods}

\section{Participants}

Fifty-three men, aged between 42 and 76 years, with diagnosed LVDD and who underwent a recent coronary artery bypass grafting procedure at the Quebec Heart and Lung Institute were prospectively recruited. All selected patients had CAD that required a coronary artery bypass grafting procedure according to current ACC/AHA practice guidelines. ${ }^{13}$ Exclusion criteria included greater than moderate LV systolic dysfunction (LV ejection fraction $<40 \%$ ), impaired renal function (creatinine $\geq 150 \mathrm{mM}$ ), known autoimmune disease, and/or lung disease and active smoking. Patients with a significant cardiac valvular disease that may eventually require surgery were also excluded. T2DM and glucose intolerance status were determined in accordance with the American Diabetes Association guidelines. ${ }^{14,15}$ Prediabetes was defined by the presence of impaired fasting glucose and/or impaired glucose tolerance. ${ }^{15} \mathrm{We}$ included patients in our study with at least stage 2 systemic hypertension diagnosed as values $>140 \mathrm{mmHg}$ for systolic blood pressure and/or $>90 \mathrm{mmHg}$ for diastolic blood pressure. ${ }^{16}$ Presence of the metabolic syndrome was diagnosed using the NCEP-ATPIII clinical criteria. ${ }^{17}$ The research was conducted in accordance with the Helsinki Declaration; informed written consent was obtained from all participants before their inclusion in the study, which had been approved by the Laval University and the Quebec Heart and Lung Institute (Quebec City, QC, Canada) Medical Ethics Committees.

\section{Intervention}

This study is a prospective analysis of men with CAD and abnormal LV diastolic function who completed a 1-year lifestyle modification program involving a personalized healthy eating strategy combined with physical activity counseling. ${ }^{18}$ The intervention began at least 6 weeks after the coronary artery bypass grafting procedure (86 days on average). Participants were counseled individually to improve their nutritional habits and physical activity once every 2 weeks during the first 4 months of the program, with subsequent monthly visits for the following 8 months. Each visit included an interactive session with a registered nutritionist followed by a meeting with a kinesiologist. The physical activity program aimed at achieving a minimum of 150 min of aerobic physical activity weekly at a moderateto-vigorous intensity ( $50 \%$ to $80 \%$ of maximum heart rate measured during maximal treadmill test). In addition to the aerobic training component, a strength-training program was performed. This type of training has been highly recommended in patients with prediabetes or T2DM. The personalized nutritional recommendation aimed at improving dietary quality in all patients, and included a moderate caloric restriction for overweight and obese patients (-500 kcal daily). The recommended macronutrient composition was $45 \%-50 \%$ of carbohydrates, $20 \%-25 \%$ of proteins (preferentially from fish and lean protein sources), and $25 \%-30 \%$ of lipids. Patients were also advised to reduce saturated fatty acid intake, and to decrease the consumption of food and beverages with added sugar and sodium. Anthropometrics, abdominal fat depots, cardiac fat depots, cardiac morphology and function, and metabolic risk profile variables were measured before and at 1 year after the lifestyle modification program.

\section{Anthropometrics}

Body mass index (BMI) was calculated and expressed in kilograms per square meter. Waist circumference was used as a clinical anthropometric measure according to international guidelines. ${ }^{19}$ Body weight and body composition were assessed using a bioelectrical impedance balance (Tanita TBF-350, Tokyo, Japan).

\section{Body fat distribution}

Abdominal adipose tissue (AT) volumes were measured at the level of the $\mathrm{L}_{4} \mathrm{~L}_{5}$ intervertebral disk by using MRI as previously described. ${ }^{20}$ Imaging was performed using a clinical 1.5 Tesla Philips Achieva system coupled to a body coil (Philips Health Care, Best, The Netherlands). ${ }^{20}$ Cardiac AT volumes were assessed by ECG-gated cardiac MRI as previously described. ${ }^{21}$ Intrathoracic AT volumes were also assessed by MRI with a dedicated cardiac coil (Philips Health Care). AT located between the myocardium and the pericardium was defined as epicardial fat, whereas AT located on the external surface of the parietal pericardium within the mediastinum was considered as pericardial fat.

\section{Cardiac morphology and function}

Cardiac morphology and function, including LV volumes, mass, ejection fraction, filling dynamic and diastolic function parameters, were acquired using standard steady-state free precession cine imaging at 30 phases per cardiac cycle in held end-expiration. Cardiac volume and function measurements were performed as previously described. ${ }^{22-24}$ Indexed cardiac chamber volumes and LV mass to body 
surface area were described. The LV mass to LV enddiastolic volume (EDV) (LV mass-to-volume ratio), an index of cardiac remodeling, was measured before and after the lifestyle intervention. ${ }^{25}$

\section{LV diastolic performance}

Dynamic cine images were used to manually quantify LV diastolic volume using short-axis slices. LV diastolic function was estimated by using early diastolic PFR. PFR corresponds to the maximal LV filling rate defined by maximal change in LV volume between sequential temporal phases ( $\Delta$ volume/ $\Delta$ phase). LV diastolic PFR was normalized to EDV. ${ }^{26}$ LVDD was defined by an early diastolic PFR normalized to EDV $<2.5 \mathrm{~mL} / \mathrm{s}^{27}$ The $\mathrm{LV}$ diastolic PFR measurement was also normalized for LV stroke volume (SV), with a PFR/SV $<4 \mathrm{~mL} / \mathrm{s}$ considered abnormal. Time interval between end-systole and PFR [time to peak filling rate (TPFR)] and the proportion of diastole required to recover $80 \%$ of SV (diastolic volume recovery) were also used as indices of LV diastolic function. ${ }^{28}$

\section{Blood collection and biochemical analysis}

After a 12-hr overnight fast, blood samples were collected into Vacutainer tubes containing EDTA (Miles Pharmaceuticals, Rexdale, Ontario, Canada). Plasma cholesterol and triglyceride concentrations were determined in plasma and lipoprotein fractions using a Technicon RA500 analyzer (Bayer Corporation, Tarrytown, NY). ${ }^{29}$ Highly sensitive C-reactive protein levels were measured by immunoassay (Dade Behring, Munich, Germany). ${ }^{30} \mathrm{~A}$ 75 grams oral glucose tolerance test was performed in patients without known T2DM $(n=43)$. Blood samples were taken at $0,30,45,60,90,120,150$, and $180 \mathrm{~min}$ for the analysis of plasma glucose and insulin levels. The integrated areas under the curve of plasma glucose and insulin were calculated by the trapezoid method. ${ }^{31}$ Insulin resistance was evaluated with the homeostasis model assessment of insulin resistance (HOMA-IR). ${ }^{32}$ Skeletal muscle insulin resistance was estimated using the inverse of the Matsuda Index. ${ }^{33}$

\section{Exercise capacity}

CRF was assessed using a maximal treadmill test according to a modified Bruce protocol ${ }^{34}$ on a treadmill (Trackmaster, Newton, KS) linked to a QuarkB2 monitor (Cosmed, Rome, Italy). During the exercise test, gas exchange was measured to determine peak oxygen consumption $\left(\mathrm{VO}_{2 \text { peak }}\right)$ normalized to body fat mass. $\mathrm{VO}_{2 \text { peak }}$ normalized to body fat mass $\left(\mathrm{mLO}_{2} / \mathrm{kg} / \mathrm{min}\right)$ was the variable used in the assessment of exercise capacity (CRF). The maximal treadmill test was conducted before and after the 1year intervention.

\section{Statistical analyses}

All data are presented as mean \pm standard deviation unless otherwise specified. Normality of data distribution was verified using normality tests (Shapiro-Wilk, KolmogorovSmirnov), skewness and kurtosis statistics. Categorical data and proportions were analyzed using the chi-squared test, the Fisher exact test, and the McNemar test. A paired $t$-test was used to compare pre/postintervention findings in the entire group and within each group. Groups were first defined according to their glucose tolerance status before the lifestyle intervention (normal glucose tolerance, prediabetes, or T2DM). Comparisons between groups were made by using ANOVA with the general linear model procedure. The Tukey test was used in situations in which a significant group effect was observed. Groups were further defined according to the diastolic function response (improved LVDD or worsened LVDD) after the 1-year lifestyle intervention. Unpaired Student's t-test was used to determine the significance of the difference between groups for normally distributed continuous variables at baseline and after the 1-year intervention. Otherwise, the Mann-Whitney U test was used. The effect of group and time (baseline vs. 1-year follow-up) was tested using the repeated-measures ANOVA for fixed effects (for normally distributed variables) and Friedman's test (for not normally distributed variables). Post hoc comparisons between treatment groups, when necessary, were performed by using the Holm-Sidak post hoc test. Associations between targeted variables, exercise capacity, diastolic function, and changes in exercise capacity, and diastolic function were carried out using Pearson's or Spearman's correlations, as appropriate. Multivariate linear regression models were used to assess predictors of diastolic function improvement. Variables entered in these models were those with clinical relevance and/or with a $P$ value $<0.1$ after a univariate analysis. The variables entered in the multivariable models were age, diabetes mellitus, and baseline BMI, visceral AT levels, insulin sensitivity, systolic BP, and exercise capacity $\left(\mathrm{VO}_{2 \text { peak }}\right.$ and exercise duration). Diastolic function severity and cardiac AT levels were also included in the second and third models, respectively. In a multiple regression model, the effects of changes in BMI, visceral AT levels, cardiac AT levels, insulin sensitivity, exercise capacity, and cardiac remodeling index on diastolic function improvement were included. The source of variation in diastolic function was calculated using the type III sum of squares. This sum of squares applies to unbalanced study designs and quantifies the effects of an independent variable after adjustment for all other variables included in the model. Statistical significance was set at two-sided $P<0.05$. Data were analyzed using the SAS software version 9.4 (SAS Institute, Inc., Cary, NC).

\section{Results}

\section{Participant characteristics}

Fifty-three men $\left(62 \pm 8\right.$ years; BMI: $\left.27.3 \pm 3.5 \mathrm{~kg} / \mathrm{m}^{2}\right)$ with CAD and LVDD (PFR normalized to EDV ratio $<2.5 \mathrm{~mL} / \mathrm{ms}$ ) were studied. The proportions of systemic hypertension, prediabetes, and T2DM were $76 \%, 43 \%$, and $26 \%$, respectively. Mean HbA1c was $6.3 \%$ among patients with T2DM. Patients had an average CRF of $24.9 \mathrm{mLO}_{2} /$ $\mathrm{kg} / \mathrm{min}$ (range: $16.3-34.4 \mathrm{mLO}_{2} / \mathrm{kg} / \mathrm{min}$ ) and a reduced PFR normalized to EDV ratio of $1.8 \mathrm{~mL} / \mathrm{ms}$ before the lifestyle modification program (Tables 1 and 2). At baseline, exercise capacity (CRF) was reduced, and LV diastolic function impaired in patients with T2DM compared with those with a normal glucose tolerance (Fig. 1 and Supplementary Tables S1 and S2). 
Table 1. Clinical Characteristics of Participants

\begin{tabular}{|c|c|c|c|}
\hline & \multicolumn{2}{|c|}{ Abnormal diastolic function } & \multirow[b]{2}{*}{$\mathrm{P}$ value } \\
\hline & Baseline & 1 year & \\
\hline$N$ & 53 & 53 & \\
\hline Age, years & $63 \pm 8$ & $64 \pm 8$ & - \\
\hline \multicolumn{4}{|l|}{ Co-morbidities $(n, \%)$} \\
\hline Systemic hypertension & $39(76)$ & - & - \\
\hline Prediabetes & $23(43)$ & - & - \\
\hline Type 2 diabetes mellitus & $14(26)$ & - & - \\
\hline Obesity (BMI $\geq 30 \mathrm{~kg} / \mathrm{m}^{2}$ ) & $12(23)$ & - & - \\
\hline Metabolic syndrome & $30(57)$ & - & - \\
\hline \multicolumn{4}{|l|}{ Anthropometrics } \\
\hline Body weight, kg & $82.9 \pm 11.7$ & $81.5 \pm 10.9$ & 0.04 \\
\hline BMI, $\mathrm{kg} / \mathrm{m}^{2}$ & $27.6 \pm 3.6$ & $27.2 \pm 3.4$ & 0.04 \\
\hline Waist circumference, $\mathrm{cm}$ & $100.3 \pm 9.8$ & $97.9 \pm 8.2$ & 0.001 \\
\hline Fat mass, kg & $20.9 \pm 6.9$ & $21.1 \pm 6.4$ & 0.53 \\
\hline Fat-free mass, kg & $61.8 \pm 6.1$ & $60.5 \pm 5.9$ & $<0.0001$ \\
\hline Visceral AT, $\mathrm{cm}^{3}$ & $194 \pm 79$ & $177 \pm 74$ & 0.04 \\
\hline Subcutaneous AT, $\mathrm{cm}^{3}$ & $215 \pm 73$ & $197 \pm 68$ & 0.001 \\
\hline Pericardial AT, $\mathrm{cm}^{3}$ & $16 \pm 9$ & $11 \pm 7$ & $<0.0001$ \\
\hline Epicardial AT, $\mathrm{cm}^{3}$ & $15 \pm 4$ & $13 \pm 3$ & $<0.0001$ \\
\hline \multicolumn{4}{|l|}{ Biochemical parameters } \\
\hline Fasting glucose, mM & $5.8 \pm 0.8$ & $5.8 \pm 0.9$ & 0.10 \\
\hline 2 -h glucose, mM & $7.9 \pm 2.1$ & $7.3 \pm 2.3$ & 0.02 \\
\hline HOMA-IR index & $2.9 \pm 1.7$ & $2.5 \pm 1.4$ & 0.04 \\
\hline 1/Matsuda index & $0.16 \pm 0.02$ & $0.14 \pm 0.02$ & 0.52 \\
\hline LDL-cholesterol, mM & $1.6 \pm 0.4$ & $1.6 \pm 0.4$ & 0.19 \\
\hline HDL-cholesterol, mM & $1.2 \pm 0.3$ & $1.3 \pm 0.3$ & 0.0005 \\
\hline Triglycerides, mM & $1.3 \pm 0.6$ & $1.2 \pm 0.5$ & $<0.0001$ \\
\hline Hs-CRP, mg/L & $2.5 \pm 3.1$ & $1.5 \pm 1.9$ & 0.02 \\
\hline \multicolumn{4}{|l|}{ Exercise testing parameters } \\
\hline Exercise duration, $\mathrm{s}$ & $611 \pm 86$ & $691 \pm 89$ & $<0.0001$ \\
\hline Cardiorespiratory fitness, $\mathrm{mL} \mathrm{O}_{2} / \mathrm{kg} / \mathrm{min}$ & $24.9 \pm 4.1$ & $28.2 \pm 4.8$ & $<0.0001$ \\
\hline \multicolumn{4}{|l|}{ Medications $(n, \%)$} \\
\hline Beta-blockers & $15(29)$ & $45(88)$ & \\
\hline Calcium channel blockers & $8(16)$ & $13(25)$ & \\
\hline ACEI/ARB & $17(32)$ & $31(58)$ & \\
\hline Diuretics & $4(8)$ & $10(20)$ & \\
\hline$\geq 3$ Antihypertensive agents & $4(8)$ & $13(25)$ & \\
\hline
\end{tabular}

Data are expressed as mean $\pm \mathrm{SD}$ or as number (percentage).

BMI, body mass index; AT, adipose tissue; HOMA-IR, homeostasis model assessment of insulin resistance; IR, insulin resistance; Hs-CRP, high-sensitive C-reactive protein; ACEI, angiotensin-converting enzyme inhibitor; ARB, angiotensin receptor blocker.

\section{Effect of a lifestyle modification program on exercise capacity and LV diastolic function}

A 1-year lifestyle modification program was associated with reductions in BMI $\left(0.4 \mathrm{~kg} / \mathrm{m}^{2}, P=0.04\right)$, waist circumference $(2.4 \mathrm{~cm}, P=0.001)$, abdominal visceral and cardiac AT levels $\left(16.7 \mathrm{~cm}^{3}, P=0.04\right.$ and $\left.7.0 \mathrm{~cm}^{3}, \quad P<0.0001\right)$ (Table 1), as well as with improvements in several parameters of the metabolic risk profile (Table 1). CRF increased by $13 \%$ after the lifestyle intervention $(P<0.0001)$. In addition, LV stroke volume increased significantly $(P=0.01)$, and cardiac remodeling index (LV mass-to-volume ratio) improved considerably $(P=0.0005)$. In the entire group, the LV diastolic function (PFR) was not significantly improved in response to the intervention $(P=0.11)$ (Table 2). However, analysis of individual response showed that $53 \%$ of patients improved LV diastolic function in response to the lifestyle modification program. Similar results were observed when
PFR normalized to LV stroke volume (PFR/SV $<4 \mathrm{~mL} / \mathrm{s}$ ) was used as criteria for LVDD.

\section{Effect of a lifestyle modification program on exercise capacity and $L V$ diastolic function according to glucose tolerance status}

At baseline, individuals with T2DM were characterized by a greater global adiposity, and higher cardiac and visceral AT levels in comparison with those with a normal glucose tolerance; a 1-year lifestyle modification program was associated with greater reductions in body weight, cardiac and visceral AT levels in patients with T2DM (Fig. 1 and Supplementary Table S1). Glycemic control was similar pre/post lifestyle intervention. Despite worsened exercise capacity before the intervention, the magnitude of CRF improvement in response to the lifestyle modification program 
Table 2. Cardiac Magnetic Resonance Measurements

\begin{tabular}{lcc}
\hline & \multicolumn{2}{c}{ Abnormal diastolic function } \\
\cline { 2 - 3 } & Baseline & 1 year \\
\hline$N$ & 53 & 53 \\
Resting HR, bpm & $62 \pm 9$ & $60 \pm 9$ \\
Systolic BP, mm Hg & $117 \pm 12$ & $117 \pm 9$ \\
Diastolic BP, mm Hg & $72 \pm 8$ & $71 \pm 7$ \\
Cardiac structure and function & & $164 \pm 33$ \\
LV end-diastolic volume, mL & $156 \pm 34$ & $62 \pm 20$ \\
LV end-systolic volume, mL & $59 \pm 20$ & $52 \pm 8$ \\
LV stroke volume/BSA, mL/m ${ }^{2}$ & $49 \pm 10$ & $62 \pm 7$ \\
LV ejection fraction, \% & $62 \pm 7$ & $102 \pm 23$ \\
LV mass, g & $105 \pm 20$ & $52 \pm 10$ \\
LV mass index, g/m & $53 \pm 9$ & $0.64 \pm 0.14$ \\
LV mass to LV end-diastolic volume, g/mL & $0.69 \pm 0.14$ & $102 \pm 24$ \\
LA end-diastolic volume, mL & $97 \pm 26$ & $52 \pm 12$ \\
LA end-diastolic volume index, mL/m ${ }^{2}$ & $49 \pm 12$ & $0.31 \pm 0.11$ \\
Peak filling rate, mL/ms & $0.29 \pm 0.10$ & $1.9 \pm 0.6$ \\
Peak filling rate to LV end-diastolic volume & $1.8 \pm 0.4$ & $3.1 \pm 1.0$ \\
Peak filling rate to LV stroke volume & $2.9 \pm 0.7$ & $208 \pm 54$ \\
Time to peak filling rate, ms & $192 \pm 51$ & $475 \pm 114$ \\
Diastolic volume recovery, ms & $417 \pm 103$ & 0.004 \\
\hline
\end{tabular}

Data are expressed as mean \pm SD.

HR, heart rate; BP, blood pressure; LV, left ventricular; BSA, body surface area; LA, left atrial.

was comparable between participants with T2DM and those with a normal glucose tolerance (Fig. 1 and Supplementary Table S1). Improvement in LV diastolic function after 1-year lifestyle modification program did not reach statistical significance in patients with T2DM (Fig. 1 and Supplementary Table S2). Patients with diagnosed prediabetes showed the greatest improvement in exercise capacity (20\% increased $\mathrm{CRF}$ ) and insulin sensitivity, whereas improvement in LV diastolic function was comparable with other groups (Fig. 1 and Supplementary Tables S1 and S2). Metabolic syndrome phenotype was not associated with a worsened LVDD (data not shown).

\section{Analysis according to $L V$ diastolic function response}

To further explore the potential clinical, metabolic, and cardiac determinants of $\mathrm{LV}$ diastolic function response after the 1-year lifestyle modification program among men with LVDD and CAD, patients who experienced LVDD improvement postlifestyle intervention were compared with those who worsened LVDD (Fig. 2 and Supplementary Table S3). Both groups had similar age and comorbidities (Supplementary Table S3). After the 1-year lifestyle modification program, patients had comparable percentage of weight loss, reductions in visceral AT levels, and improvement in exercise capacity (Fig. 2 and Supplementary Table S3). Compared with those who worsened LVDD, participants who responded to the lifestyle modification program by improving LVDD showed a significant reduction in resting $\mathrm{HR}(P=0.04)$, significant improvements in LV stroke volume $(P<0.0001)$, and cardiac remodeling index $(P<0.0001)$ (Fig. 2). Patients in the lowest LVDD tertile (PFR-to-EDV ratio $<1.64 \mathrm{~mL} / \mathrm{s}$ ), characterized by the worsened LV diastolic function at baseline, experienced comparable improvement in LV diastolic function and exercise capacity than other tertiles (data not shown). No association between diastolic function severity before the intervention and extent of diastolic function improvement after the lifestyle intervention was found.

\section{Correlates of $L V$ diastolic function improvement}

Age was negatively correlated with LV diastolic function $(R=-0.50, P=0.0001)$. No significant association was found between changes in LV diastolic function and percentage of weight loss, visceral and cardiac AT levels, insulin sensitivity, and exercise capacity $\left(\mathrm{VO}_{2 \text { peak }}\right)$. Multivariable regression analysis, including baseline characteristics such as age, diabetes status, BMI, visceral AT, insulin sensitivity, systolic blood pressure, and exercise capacity $\left(\mathrm{VO}_{2 \text { peak }}\right)$ in a model, showed that only age (partial $R^{2}=26.9, P<0.0001$ ) and diabetes (partial $R^{2}=5.9, P=0.04$ ) are predictors of change in $\mathrm{LV}$ diastolic function. Change in cardiac remodeling index emerged as the only independent correlate of improvement in $\mathrm{LV}$ diastolic function after the lifestyle intervention $\left(R^{2}=21.9\right.$, $P=0.002)$.

\section{Discussion}

The main findings of this study are as follows: (1) a 1year exercise training-based lifestyle modification program in addition to standard care in men with LVDD and CAD was associated with significant benefits on exercise capacity; (2) half of patients with varying degrees of glucose tolerance improved LV diastolic function after the lifestyle modification program; among them, $13 \%$ normalized LV diastolic function; and (3) despite a worse exercise capacity and LV diastolic function before the lifestyle intervention, patients with T2DM showed comparable benefits from exercise training-based lifestyle intervention with those without T2DM. 

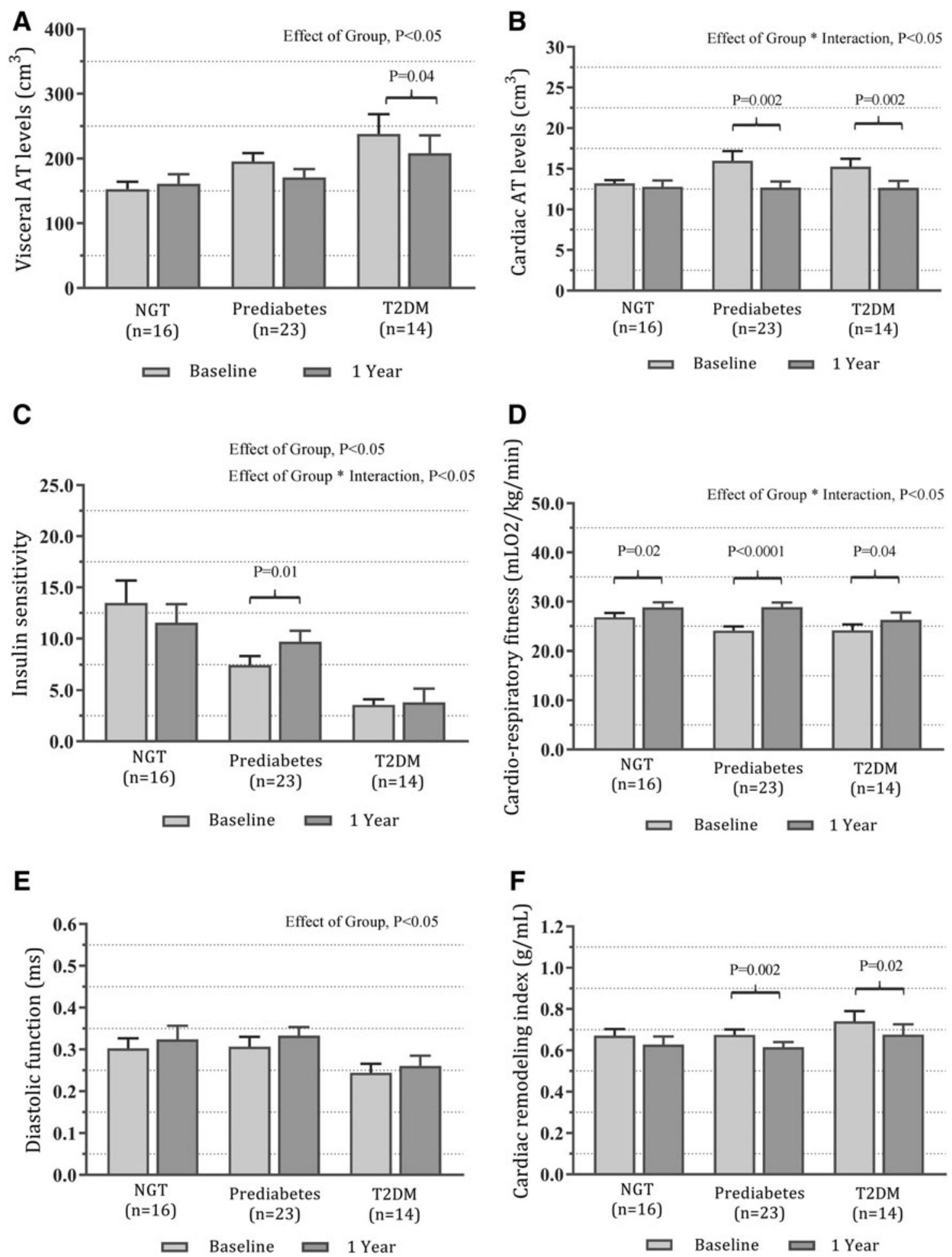

FIG. 1. Visceral adipose tissue levels (A), epicardial adipose tissue levels (B), insulin sensitivity (Matsuda index) (C), exercise capacity (cardiorespiratory fitness) (D), diastolic function-peak filling rate (E), and LV remodeling index (LV mass-to-volume ratio) (F) in men with abnormal diastolic function and coronary artery disease according to glucose tolerance status, before and 1 year after exercise training-based lifestyle intervention. Data are presented as mean with SEM. Light gray bar, preintervention; dark gray bar, postintervention. AT, adipose tissue; NGT, normal glucose tolerance; T2DM, type 2 diabetes mellitus; LV, left ventricular. 

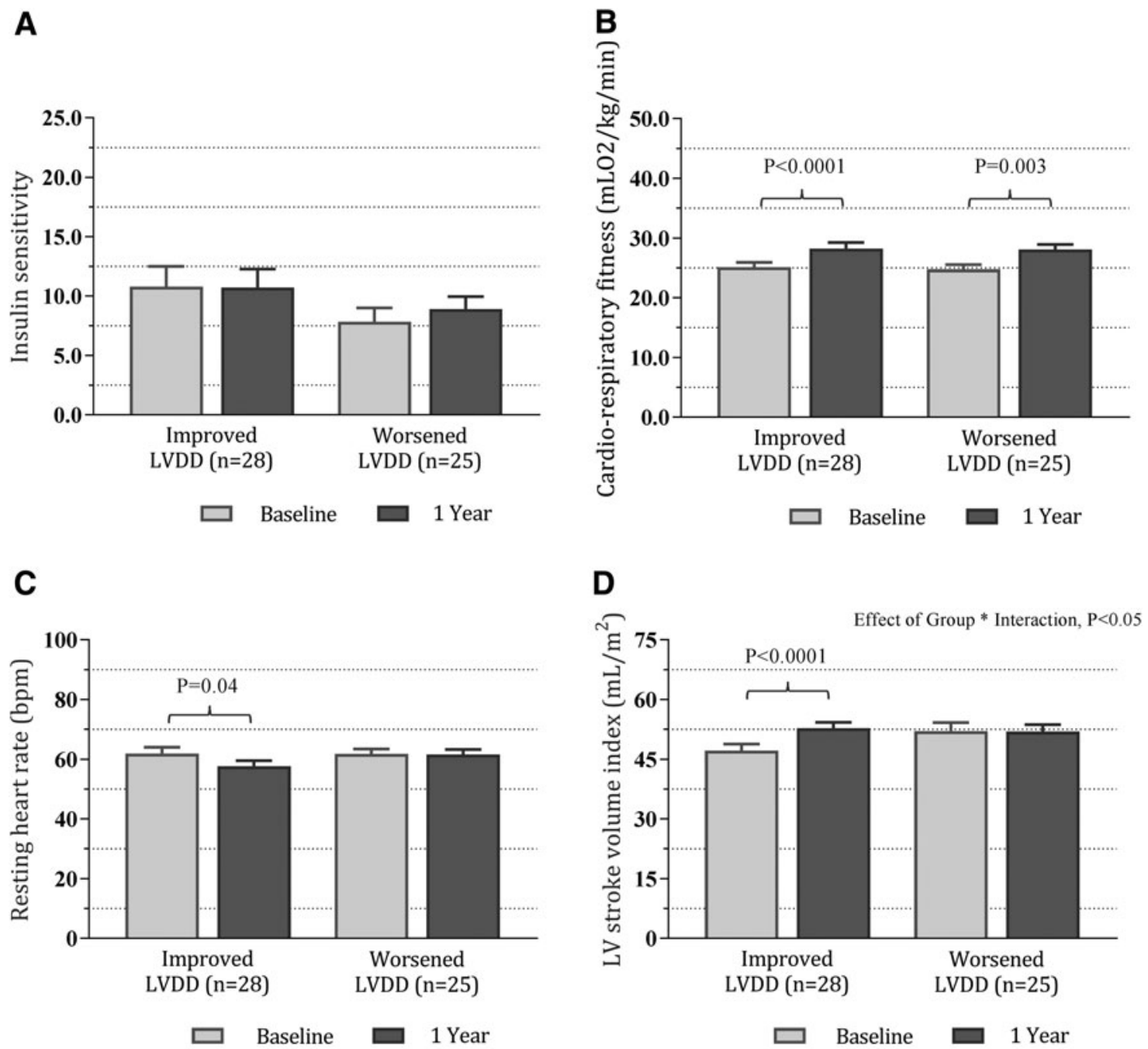

$\mathbf{E}$

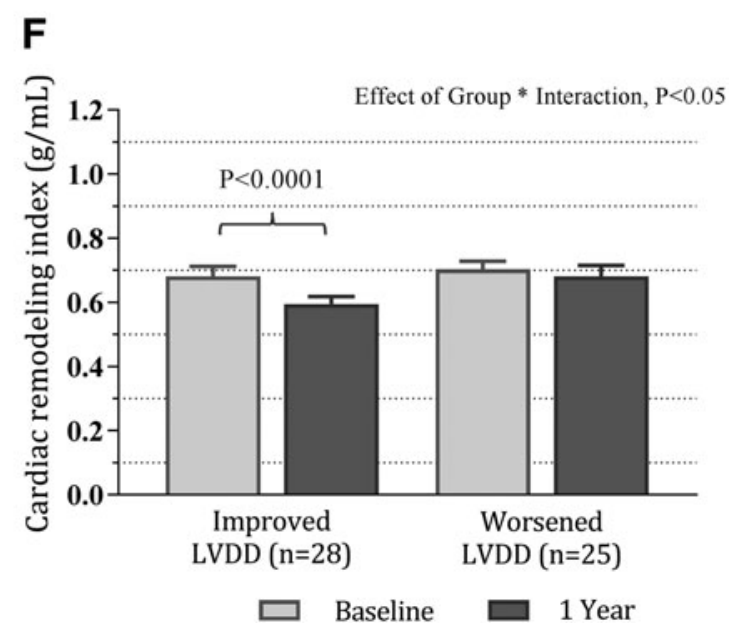

FIG. 2. Insulin sensitivity (Matsuda index) (A), exercise capacity (cardiorespiratory fitness) (B), resting heart rate (C), LV stroke volume index (D), LV mass (E), and LV remodeling index (LV mass-to-volume ratio) (F) according to LVDD response before and 1 year after exercise training-based lifestyle intervention. Data are presented as mean with SEM. Light gray bar, preintervention; dark gray bar, postintervention. LVDD, left ventricular diastolic dysfunction. 


\section{Effects of the lifestyle modification intervention}

In line with our data, others have demonstrated that exercise training associated with comparable CRF improvements $\left(9 \%-13 \% \quad \mathrm{VO}_{2 \text { peak }}\right)$ had benefits on cardiac performance and LV diastolic function in different populations, ${ }^{6,35,36}$ and in some cases, normalized LV diastolic function in individuals with well-controlled T2DM. ${ }^{6}$ To the best of our knowledge, although we have not been able to explain the mechanisms behind such an adaptation, this is the first study to show the positive impact of a 1-year lifestyle modification program in addition to standard care on both exercise capacity and LV diastolic function in men with LVDD and CAD, with varying levels of glucose tolerance using a detailed analysis of cardiac performance and LV diastolic function, coupled with maximal exercise capacity measures.

Exercise training-based lifestyle modification program in our population also results in favorable cardiac remodeling and impact on cardiac performance. These observations are of clinical importance since patients with T2DM and CAD have considerable reduced cardiac contractile capacities. These results are in line with works of Cassidy et al. who observed a significant increase in LV stroke volume accompanied by LV diastolic function improvement in patients with T2DM and without CAD. ${ }^{37}$ Benefits of exercise training on LV diastolic function and on cardiac remodeling in CAD may be due to multiple mechanisms involving improvement in endothelial function, reduction in systemic vascular resistance, reduced preload, adjustment in autonomic system, and reduction in resting $\mathrm{HR}$ and blood pressure. $^{7}$ Cardiovascular drugs also have favorable cardiac remodeling properties, ${ }^{38}$ and might have influenced our findings. After a CABG procedure, patients with T2DM were reported to have a lower exercise capacity and higher prevalence and severity of LVDD. ${ }^{39}$ In this study, exercise capacity and parameters of LV diastolic function were impaired in T2DM patients compared with patients with a normal glucose tolerance. After the lifestyle modification program, T2DM patients improved these parameters to a similar extent as their normal glucose tolerance counterparts. No relationship with glycemic control was found. As LVDD is a prevalent condition in patients with T2DM, and an independent predictor of functional capacity and mortality, this emphasized the clinical relevance of these findings.

\section{Lifestyle modification intervention and $L V$ diastolic function response}

Although exercise capacity was improved after the lifestyle modification program, LV diastolic function response to intervention has been found to be heterogeneous. Analysis of individual LV diastolic function response to lifestyle modification program showed that $53 \%$ of patients engaged in the lifestyle intervention improved LV diastolic function. Similar findings have been reported in men with metabolic syndrome. ${ }^{35}$ Improvement in LV diastolic function after a lifestyle modification program in patients with CAD may be influenced by multiple factors, including enhanced exercise program, exercise training type, presence of residual myocardial ischemia postrevascularization, cardiac fibrosis, LV diastolic function severity, changes in cardiac loading con- ditions, cardiac steatosis and intrinsic changes in myocardial metabolism and vascular function, ${ }^{37,40-42}$ reflecting the complexity and heterogeneity of underlying pathophysiological mechanisms of LV diastolic function in patients with CAD and varying degrees of glucose tolerance.

Although it has been proposed that LV diastolic function improvement after exercise training correlates with the magnitude of exercise capacity response, ${ }^{3,6,40}$ no association was observed between LV diastolic function improvement and the magnitude of exercise capacity response as determined by $\mathrm{VO}_{2 \text { peak }}$. The discrepancy between our findings and others may be attributable to our sample size, methods used, exercise training protocol, or difference in population studied. Similarly, Smart et al. did not report any association between parameters of $\mathrm{LV}$ diastolic function improvement and change in exercise capacity $\left(\mathrm{VO}_{2 \text { peak }}\right)$ after a 16 -week exercise-based training in patients with LVDD. ${ }^{43}$ Recently, studies indicate that exercise intensity may be an important factor in improving cardiac remodeling and LV diastolic function, with high-intensity intermittent training intervention found to be more effective than moderate-intensity endurance training at reducing cardiovascular risk factors in $\mathrm{T}_{2} \mathrm{DM}^{44}$ and at reversing cardiac remodeling in patients with postinfarction heart failure. ${ }^{45}$ In line with these findings, others have found that longer term intervention using moderate exercise aerobic training demonstrated improvement only when exercise was performed in the vigorous zone. ${ }^{46}$ These findings suggest that patients with adverse cardiac remodeling and LVDD, who are at a greater risk of nonresponse to exercise, may require alternative exercise training strategies (e.g., higher intensity and/or dose of exercise training) to improve cardiac performance and $\mathrm{LV}$ diastolic function. The physiological mechanisms responsible for these beneficial changes remain to be explored. Exercise training response may also be influenced by intrinsic favorable changes in myocardial metabolism, skeletal muscle function, and vascular function. ${ }^{47}$

In this study, weight loss that was achieved after the 1year lifestyle intervention was relatively modest. On the contrary, this 1-year lifestyle modification program has been proven sufficient to induce a significant reduction in cardiac and visceral fat depots, mostly in patients with impaired glucose tolerance and T2DM. Despite previous findings that suggested visceral and cardiac adiposity as potential modulators of LV diastolic function improvement after exercise training, ${ }^{48,49}$ we did not find significant association in our study.

\section{Strengths and limitations}

This study has used MRI-derived gold standard measurements of cardiac morphology, cardiac function as well as body fat distribution (abdominal and cardiac fat depots), coupled with direct measurement of exercise capacity. However, there are some limitations to this work. The study was performed in Caucasian men with CAD, hence limiting generalization to other populations. The noncontrolled nature of our intervention limits any causal interpretations. In addition, we did not follow a group of postcoronary artery bypass grafting patients in parallel without lifestyle modification counseling. Therefore, some favorable changes in exercise capacity and LV diastolic performance could be attributable to the postrevascularization "recovery," drug 
therapy, or natural outcomes. As improvements in exercise capacity and visceral AT levels were the primary outcomes of this intervention, it is unknown if the accompanying changes in LV diastolic performance reflect a true biologic association between these parameters, or whether improvement in LV diastolic function was only observed in men who better adhered to the lifestyle modification program. Despite these limitations, our results are still clinically significant and unique, and provide new insights and perspectives on a growing high-risk population with CAD and varying degrees of glucose tolerance.

\section{Conclusions}

A 1-year lifestyle modification program in addition to standard care in men with LVDD and CAD is associated with benefits on exercise capacity, cardiac performance, and $\mathrm{LV}$ diastolic function in half of patients. Importantly, exercise capacity and LV diastolic function have been found modifiable in high-risk patients with T2DM and CAD. Whether these positive changes in exercise capacity, cardiac remodeling, and $\mathrm{LV}$ diastolic function will translate into reduced adverse cardiovascular outcomes remains to be demonstrated by longitudinal studies.

\section{Author Contributions}

All authors contributed to the conception and/or design of the work. M.E.P., P.P., A.M., V.L., K.B., E.L., and J.P.D. contributed to the acquisition, analysis, or interpretation of data for the work. M.E.P., P.P., and J.P.D. drafted the article. All authors contributed to the interpretation of data for the work. All authors also critically revised the article, gave final approval, and agreed to be accountable for all aspects of work ensuring integrity and accuracy.

\section{Acknowledgments}

We express our gratitude to the patients of this study for their excellent collaboration. We want to specially thank the staff for their help with data collection.

This study was supported by the Canadian Institutes of Health Research (CIHR) and the Tekes Finnish Funding Agency. M.E.P., B.A., P.P., and E.L. are research scholars from the Fonds de Recherche du Québec-Santé (FRQ-S); P.M. holds a Fonds de Recherche du Québec-Santé (FRQ-S) Research Chair on the Pathobiology of Calcific Aortic Valve Disease. A.M. holds a research Chair on the pathogenesis of insulin resistance and cardiovascular diseases, which is supported by the CIHR; E.L. holds the Laval University Chair of Research \& Innovation in Cardiovascular Imaging; J.P.D. is the scientific director of the International Chair on Cardiometabolic Risk, which is supported by the CIHR.

\section{Author Disclosure Statement}

The author(s) declared no potential conflicts of interest with respect to the research, authorship, and/or publication of this article.

\section{Supplementary Material}

Supplementary Table S1

Supplementary Table S2

Supplementary Table S3

\section{References}

1. Meta-Analysis Research Group in Echocardiography AMIC, Moller JE, Whalley GA, et al. Independent prognostic importance of a restrictive left ventricular filling pattern after myocardial infarction: An individual patient meta-analysis: Meta-Analysis Research Group in Echocardiography acute myocardial infarction. Circulation 2008;117:2591-2598.

2. Barmeyer A, Mullerleile K, Mortensen K, et al. Diastolic dysfunction in exercise and its role for exercise capacity. Heart Fail Rev 2009;14:125-134.

3. Chan E, Giallauria F, Vigorito C, et al. Exercise training in heart failure patients with preserved ejection fraction: A systematic review and meta-analysis. Monaldi Arch Chest Dis 2016;86:759.

4. Heran BS, Chen JM, Ebrahim S, et al. Exercise-based cardiac rehabilitation for coronary heart disease. Cochrane Database Syst Rev 2011;1:CD001800.

5. Ades PA. Cardiac rehabilitation and secondary prevention of coronary heart disease. N Engl J Med 2001;345:892902.

6. Brassard P, Legault S, Garneau C, et al. Normalization of diastolic dysfunction in type 2 diabetics after exercise training. Med Sci Sports Exerc 2007;39:1896-1901.

7. Hill JA, Olson EN. Cardiac plasticity. N Engl J Med 2008; 358:1370-1380.

8. Rider OJ, Francis JM, Ali MK, et al. Effects of catecholamine stress on diastolic function and myocardial energetics in obesity. Circulation 2012;125:1511-1519.

9. Mendoza DD, Codella NC, Wang Y, et al. Impact of diastolic dysfunction severity on global left ventricular volumetric filling - assessment by automated segmentation of routine cine cardiovascular magnetic resonance. J Cardiovasc Magn Reson 2010;12:46.

10. Nacif MS, Almeida ALC, Young AA, et al. ThreeDimensional Volumetric Assessment of Diastolic Function by Cardiac Magnetic Resonance Imaging: The MultiEthnic Study of Atherosclerosis (MESA). Arq Bras Cardiol 2017;108:552-563.

11. Nyman K, Graner M, Pentikainen MO, et al. Cardiac steatosis and left ventricular function in men with metabolic syndrome. J Cardiovasc Magn Reson 2013;15: 103.

12. Graca B, Donato P, Ferreira MJ, et al. Left ventricular diastolic function in type 2 diabetes mellitus and the association with coronary artery calcium score: A cardiac MRI study. AJR Am J Roentgenol 2014;202:12071214.

13. Hillis LD, Smith PK, Anderson JL, et al. 2011 ACCF/AHA Guideline for Coronary Artery Bypass Graft Surgery: Executive summary: A report of the American College of Cardiology Foundation/American Heart Association Task Force on Practice Guidelines. Circulation 2011;124:2610 2642.

14. American Diabetes A. Diagnosis and classification of diabetes mellitus. Diabetes Care 2009;32 Suppl 1:S62S67.

15. American Diabetes A. 2. Classification and Diagnosis of Diabetes: Standards of Medical Care in Diabetes-2018. Diabetes Care 2018;41(Suppl 1):S13-S27.

16. Whelton PK, Carey RM, Aronow WS, et al. 2017 ACC/AHA/AAPA/ABC/ACPM/AGS/APhA/ASH/ASPC/ NMA/PCNA Guideline for the Prevention, Detection, Evaluation, and Management of High Blood Pressure in Adults: A Report of the American College of 
Cardiology/American Heart Association Task Force on Clinical Practice Guidelines. J Am Coll Cardiol 2018;71: e127-e248.

17. Executive Summary of The Third Report of The National Cholesterol Education Program (NCEP) Expert Panel on Detection, Evaluation, And Treatment of High Blood Cholesterol In Adults (Adult Treatment Panel III). JAMA 2001;285:2486-2497.

18. Borel AL, Nazare JA, Baillot A, et al. Cardiometabolic risk improvement in response to a 3-yr lifestyle modification program in men: Contribution of improved cardiorespiratory fitness vs. weight loss. Am J Physiol Endocrinol Metab 2017;312:E273-E281.

19. van der Kooy K, Seidell JC. Techniques for the measurement of visceral fat: A practical guide. Int J Obes Relat Metab Disord 1993;17:187-196.

20. Boyer M, Levesque V, Poirier P, et al. Impact of a 1-year lifestyle modification program on plasma lipoprotein and PCSK9 concentrations in patients with coronary artery disease. J Clin Lipidol 2016;10:1353-1361.

21. De Larochelliere E, Cote J, Gilbert G, et al. Visceral/epicardial adiposity in nonobese and apparently healthy young adults: Association with the cardiometabolic profile. Atherosclerosis 2014;234:23-29.

22. Cerqueira MD, Weissman NJ, Dilsizian V, et al. Standardized myocardial segmentation and nomenclature for tomographic imaging of the heart: A statement for healthcare professionals from the Cardiac Imaging Committee of the Council on Clinical Cardiology of the American Heart Association. Circulation 2002;105:539-542.

23. Schulz-Menger J, Bluemke DA, Bremerich J, et al. Standardized image interpretation and post processing in cardiovascular magnetic resonance: Society for Cardiovascular Magnetic Resonance (SCMR) board of trustees task force on standardized post processing. J Cardiovasc Magn Reson 2013;15:35.

24. Le Ven F, Bibeau K, De Larochelliere E, et al. Cardiac morphology and function reference values derived from a large subset of healthy young Caucasian adults by magnetic resonance imaging. Eur Heart J Cardiovasc Imaging 2016; 17:981-990.

25. Rodriguez CJ, Diez-Roux AV, Moran A, et al. Left ventricular mass and ventricular remodeling among Hispanic subgroups compared with non-Hispanic blacks and whites: MESA (Multi-ethnic Study of Atherosclerosis). J Am Coll Cardiol 2010;55:234-242.

26. Zeidan Z, Erbel R, Barkhausen J, et al. Analysis of global systolic and diastolic left ventricular performance using volume-time curves by real-time three-dimensional echocardiography. J Am Soc Echocardiogr 2003;16: 29-37.

27. Mancini GB, Slutsky RA, Norris SL, et al. Radionuclide analysis of peak filling rate, filling fraction, and time to peak filling rate. Response to supine bicycle exercise in normal subjects and patients with coronary disease. Am J Cardiol 1983;51:43-51.

28. Kawaji K, Codella NC, Prince MR, et al. Automated segmentation of routine clinical cardiac magnetic resonance imaging for assessment of left ventricular diastolic dysfunction. Circ Cardiovasc Imaging 2009;2: 476-484.

29. Allain CC, Poon LS, Chan CSG, et al. Enzymatic determination of total serum cholesterol. Clin Chem 1974;20: $470-475$.
30. Pearson TA, Mensah GA, Alexander RW, et al. Markers of inflammation and cardiovascular disease: Application to clinical and public health practice: A statement for healthcare professionals from the Centers for Disease Control and Prevention and the American Heart Association. Circulation 2003;107:499-511.

31. Le Floch JP, Escuyer P, Baudin E, et al. Blood glucose area under the curve. Methodological aspects. Diabetes Care 1990;13:172-175.

32. Matthews DR, Hosker JP, Rudenski AS, et al. Homeostasis model assessment: Insulin resistance and beta-cell function from fasting plasma glucose and insulin concentrations in man. Diabetologia 1985;28:412-419.

33. Matsuda M, DeFronzo RA. Insulin sensitivity indices obtained from oral glucose tolerance testing: Comparison with the euglycemic insulin clamp. Diabetes Care 1999;22: 1462-1470.

34. Poirier P, Garneau C, Bogaty $\mathrm{P}$, et al. Impact of left ventricular diastolic dysfunction on maximal treadmill performance in normotensive subjects with wellcontrolled type 2 diabetes mellitus. Am J Cardiol 2000;85: 473-477.

35. Leclerc J, Arsenault M, Despres JP, et al. Determinants of Improvement In Left Ventricular Diastolic Function Following a 1-Year Lifestyle Modification Program in Abdominally Obese Men with Features of the Metabolic Syndrome. Metab Syndr Relat Disord 2016;14: 483-491.

36. Yu CM, Li LS, Lam MF, et al. Effect of a cardiac rehabilitation program on left ventricular diastolic function and its relationship to exercise capacity in patients with coronary heart disease: Experience from a randomized, controlled study. Am Heart J 2004;147:e24.

37. Cassidy S, Thoma C, Hallsworth K, et al. High intensity intermittent exercise improves cardiac structure and function and reduces liver fat in patients with type 2 diabetes: A randomised controlled trial. Diabetologia 2016; 59:56-66.

38. Cohn JN, Ferrari R, Sharpe N. Cardiac remodelingconcepts and clinical implications: A consensus paper from an international forum on cardiac remodeling. Behalf of an International Forum on Cardiac Remodeling. J Am Coll Cardiol 2000;35:569-582.

39. Wu YW, Hsu CL, Wang SS, et al. Impaired exercise capacity in diabetic patients after coronary bypass surgery: Effects of diastolic and endothelial function. Cardiology 2008;110:191-198.

40. Smart N, Haluska B, Jeffriess L, et al. Exercise training in systolic and diastolic dysfunction: Effects on cardiac function, functional capacity, and quality of life. Am Heart J 2007;153:530-536.

41. Rayner JJ, Banerjee R, Holloway CJ, et al. The relative contribution of metabolic and structural abnormalities to diastolic dysfunction in obesity. Int J Obes (Lond) 2018;42: 441-447.

42. Levelt E, Mahmod M, Piechnik SK, et al. Relationship Between Left Ventricular Structural and Metabolic Remodeling in Type 2 Diabetes. Diabetes 2016;65:44-52.

43. Smart N, Haluska B, Jeffriess L, et al. Cardiac contributions to exercise training responses in patients with chronic heart failure: A strain imaging study. Echocardiography 2006;23:376-382.

44. Pandey A, Suskin N, Poirier P. The Impact of Burst Exercise on Cardiometabolic Status of Patients Newly 
Diagnosed With Type 2 Diabetes. Can J Cardiol 2017;33: 1645-1651.

45. Hollekim-Strand SM, Bjorgaas MR, Albrektsen G, et al. High-intensity interval exercise effectively improves cardiac function in patients with type 2 diabetes mellitus and diastolic dysfunction: A randomized controlled trial. J Am Coll Cardiol 2014;64:1758-1760.

46. Hordern MD, Coombes JS, Cooney LM, et al. Effects of exercise intervention on myocardial function in type 2 diabetes. Heart 2009;95:1343-1349.

47. Marwick TH, Wong CY. Role of exercise and metabolism in heart failure with normal ejection fraction. Prog Cardiovasc Dis 2007;49:263-274.

48. Levelt E, Pavlides M, Banerjee R, et al. Ectopic and Visceral Fat Deposition in Lean and Obese Patients With Type 2 Diabetes. J Am Coll Cardiol 2016;68:53-63.
49. Rijzewijk LJ, van der Meer RW, Smit JW, et al. Myocardial steatosis is an independent predictor of diastolic dysfunction in type 2 diabetes mellitus. J Am Coll Cardiol 2008;52:1793-1799.

Address correspondence to: Jean-Pierre Després, PhD, FAHA, FIAS

Department of Cardiology

Quebec Heart and Lung Institute 2725 Chemin Sainte-Foy Quebec G1V 4G5

Canada

E-mail: jean-pierre.despres@criucpq.ulaval.ca 